Miriam Meckel

\title{
Das Internet
}

Medienevolution oder Medienrevolution?1

Jede neue Medientechnologie löst ebenso Heilsversprechen wie Horrorszenarien aus. Prognosen zur Zukunft einer vernetzten Welt, die im Wesentlichen auf der Plattform Internet basieren wird, beinhalten ebenfalls alle Ausprägungen einer nach oben und unten offenen Bewertungsskala. Dies betrifft die Konsequenzen des Netzes bzw. der Netzwerktechnologie und ihre Auswirkungen auf das politische, wirtschaftliche und soziale Leben in einer vernetzten Welt, vor allem aber die Neuordnungen im Medienensemble. Dass man mit solchen Prognosen bedachtsam umgehen muss, erschließt sich aus einer kulturgeschichtlichen Retrospektive der jeweils zeitgebundenen Bewertungen neuer Technologien und ihrer Zukunftsoptionen. So prognostizierte Thomas Watson, Chairman von IBM, 1943: „I think there is a world market for maybe five computers." Ähnlich skeptische Bewertungen der Massenvermarktbarkeit und gesellschaftlichen Implementierungsfähigkeit von Computern haben auch andere Experten abgegeben. "There is no reason anyone would want a computer in their home", resümierte Ken Olson, Gründer und Chairman von DEC, 1977, und selbst Bill Gates, Gründer und Chef von Microsoft, war noch 1981 der Ansicht: "640 K ought to be enough for anybody."

Prognosen zur Entwicklungsdynamik und gesellschaftlichen Akzep$\tan z$ von neuen Technologien sind immer kontingent. Das bedeutet allerdings nicht gleichzeitig, dass sie auch sinn- oder nutzlos wären. Vielmehr induzieren Systematisierung und Analyse von Zukunftsprozessen jeweils Rückkopplungseffekte für die Gegenwart, die dann wiederum dafür verantwortlich sind, dass zukünftige Entwicklungen durch Jetztentscheidungen beeinflusst werden - ein Phänomen, das die Prognostik als „Interventionsparadoxien" bezeichnet. Diese Zusammenhänge sind auch für die Frage nach den Konsequenzen einer zunehmenden Verbreitung und Nutzung des Internet für die traditionellen Medien von großer Bedeutung. Haben doch die typischen Verdrängungsszenarien, die bei jedem neuen Medium, bei jeder neuen Technologie existenzbedrohende Konsequenzen für bestehende Medien und Technologien befürchten, zu einer erheblichen Sensibilisierung der traditionellen Medien gegenüber dem Internet geführt. Wenn Bill Gates also Ende der 90er Jahre das Aus für

1 Der Aufsatz wurde als Vortrag anlässlich der Mitgliederversammlung des Fachverbandes Konfessionelle Presse im Verband Deutscher Zeitschriftenverleger (VDZ) am 15. November 2000 in Berlin gehalten. 
traditionelle Medienwirtschaftszweige verkündet hat ("Das Jahr 2000 wird das Ende der Zeitungs- und Zeitschriften-Verleger einläuten“), so handelt es sich dabei gewiss nicht um eine selbsterfüllende Prophezeiung, sondern um einen "Warnschuss", der bei den traditionellen Medienanbietern zu einer höheren Sensibilität und stärkeren Auseinandersetzung mit den Potentialen des Internet geführt hat.

\section{Wachstums- und Entwicklungspotentiale des Internet}

In vielerlei Hinsicht lassen sich die Anzeichen der Internetentwicklung inzwischen so deuten, dass das Netz unser bestehendes Medienensemble nicht revolutioniert, sondern vielmehr die jeweiligen Kernkompetenzen einzelner Medien akzentuiert und dabei als Katalysator wirkt. Eine entsprechende Entwicklungsdynamik zeichnet sich seit Ende der 90er Jahre $\mathrm{ab}$, die eigentlich spannende Phase steht allerdings in den kommenden drei bis fünf Jahren erst bevor: Während im Jahr 2000 knapp 300 Millionen Menschen weltweit das Internet nutzten, sollen es im Jahre 2005 mehr als 700 Millionen sein. Die Chancen, die sich aus dieser Entwicklungsdynamik ergeben, hängen letztlich davon $a b$, ob und wie es gelingt, nationale Gesellschaften und Märkte anschlussfähig zu halten. In dieser Hinsicht weist Deutschland durchaus noch Entwicklungspotentiale auf: Während in den USA und in den nordeuropäischen Ländern (Dänemark, Finnland, Schweden) eine Anschlussdichte von weit mehr als 50 Prozent der Bevölkerung erreicht ist, sind es in Deutschland etwa 37 Prozent (manche Statistiken arbeiten auch mit weit niedrigeren Zahlen), die anschlussfähig sind und das Internet nutzen.

Inwieweit es gelingt, das Netz auf eine breite gesellschaftliche Basis zu stellen, wird auch darüber entscheiden, wie sich das Medienensemble dauerhaft formieren wird und welche Nutzungsstrukturen dabei dominieren. Gerade beim Internet geht es eben nicht nur um quantitative Wachstumsprozesse, die eine generelle Anschlussfähigkeit der Gesellschaft möglich machen sollen. Vielmehr bringt das Internet auch qualitative Veränderungen mit sich, die sämtliche Lebens- und Arbeitsbereiche betreffen. Das Netz wird unsere gesellschaftlichen Strukturen verändern, sie vernetzen, dezentralisieren und einer stetigen Aktualisierbarkeit und Rekombinierbarkeit zugänglich machen.

\section{Potential 1: Die Vernetzung}

Struktur und Dynamik der Informationsverwaltung im Internet verändern langfristig gesamtgesellschaftliche Zugriffs- und Verarbeitungspro- 
zesse. Während das menschliche Informationsmanagement - unabhängig von der jeweiligen kognitiven Leistungsfähigkeit - noch immer eher linear und hierarchisch verläuft, eröffnet das Internet völlig andere Zugangswege und -arten. Ein Beispiel: Das Lesen eines Zeitungsartikels beginnt in der oberen linken Ecke des Artikels und endet in der unteren rechten Ecke. Der Text wird linear, also Zeile für Zeile erschlossen und der Rezeptionsprozess folgt einer stratifikatorischen Hierarchiebildung (das Wichtigste am Anfang, die weiteren Absätze werden unter Umständen gar nicht mehr gelesen). Im Internet ist dies anders. Hier kann der Informationssuchende, der Nutzer, an jeder beliebigen Stelle eines Web-Angebots einsteigen, sich gezielt oder auch assoziativ von Hyperlink zu Hyperlink klicken und an jeder beliebigen Stelle des Angebotes wieder aussteigen. Bei Informations- und Unterhaltungsangeboten im Netz kann man sich daher nicht darauf verlassen, dass sie als Ganzes genutzt werden, sondern sie müssen in jedem kleinen Teil, in jeder kleinteiligen sachlichen zeitlichen und sozialen Beziehung zwischen zwei Links in sich verständlich sein.

Was dies für den menschlichen Umgang mit Information bedeutet, verdeutlicht der Web-Katalog "Webbrain" (www.webbrain.com). Er vollzieht die Suche nach Informationen zu bestimmten Begriffen nach dem Gebot der ständigen Reassoziierbarkeit. Ein erster in die Suchleiste eingegebener Begriff führt nicht nur zu einer Liste der in diesem Zusammenhang interessanten Dokumente, sondern auch zu einer visualisierten Benutzeroberfläche, die eine Vielzahl von assoziierten Begriffen und Themen im Sinne eines Netzwerks um den eigentlichen Suchbegriff gruppiert. Mit der Maus lässt sich nun jeder dieser assoziierten Begriffe in den Fokus der Suche rücken und Webbrain erstellt unmittelbar ein neues Assoziationsnetzwerk, das wiederum an diesem Zentralbegriff aufgehängt wird. Durch Vernetzung wird die Informationssuche folglich nicht mehr nur auf die Flächendimension beschränkt, sondern auch die Tiefenstruktur des virtuellen Raums genutzt. Informationsanbieter und -nutzer müssen angesichts dessen umlernen: Informationsverarbeitung erfolgt unter Bedingungen der Vernetzung nicht mehr linear, sondern reflexiv, nicht mehr nach stratifikatorisch-hierarchischem, sondern assoziativ-kybernetischem Muster. Das information processing nähert sich damit ein Stück weit den Informationsverarbeitungsprozessen im menschlichen Gehirn, wird also auch erheblich komplexer.

\section{Potential 2: Ökonomie der Aufmerksamkeit}

Angesichts erweiterter Speicherkapazitäten und immer umfangreicherer Informationsangebote entsteht eine Konkurrenz um die Aufmerksamkeit 
der potentiellen Nutzerinnen und Nutzer. Aufmerksamkeit gilt heute nicht mehr nur als der Tauschwert innerhalb sozialer Beziehungen, sondern bestimmt auch zunehmend die Entstehung und Entwicklung von Märkten, die sich durch Informationen als Güter oder durch Informationen über Güter konstituieren. Aufmerksamkeit avanciert damit zum zentralen Tauschwert unserer Gesellschaft, weil öffentliche Wahrnehmbarkeit längst über Erfolg bzw. Misserfolg politischen, wirtschaftlichen oder auch sozialen bzw. individuellen Entscheidungshandelns bestimmt. Mag das Aufmerksamkeitspotential einer Gesellschaft unbeschränkt sein, so bleibt das individuelle, an bestimmte Zeitpunkte der Aufmerksamkeitszuwendung gebundene Aufmerksamkeitspotential durchaus beschränkt. Der einzelne Mensch ist nur begrenzt in der Lage, „informationelles Multitasking" zu betreiben, seine Aufmerksamkeit also zu einem bestimmten Zeitpunkt verschiedenen Angeboten zuzuwenden. Unsere Gesellschaft wird sich damit zu einer Aufmerksamkeitskonkurrenzgesellschaft entwickeln, in der Aufmerksamkeit als Tauschwert eine ähnliche Rolle spielen wird, wie das Geld sie in der industriellen Gesellschaft gespielt hat.

Berücksichtigt man allein, dass jeden Tag etwa 1,6 Millionen neue Seiten ins World Wide Web eingestellt werden, sich das Web also alle sechs Monate verdoppelt, so dürfte dies schon Indikator genug sein, um abzuleiten, dass eine Ökonomie der Aufmerksamkeit durch erhebliche Konkurrenzsituationen im Angebot von Information und Unterhaltung geprägt sein wird - im Netz ebenso wie in den traditionellen Medien.

Die englische Sprache hat diese Konzeptualisierung der "geldwerten Aufmerksamkeit" von jeher vorgesehen (,to pay attention"), während im deutschen Verständnis die Gewährung von Aufmerksamkeit eher als sozialer und humanitärer Akt verstanden wird („Aufmerksamkeit spenden").

\section{Konsequenzen und Herausforderungen für die Medienkommunikation}

Mit jedem neuen Medium, mit jeder neuen Technologie war bislang die Befürchtung verbunden, traditionelle Medien und Technologien würden durch das Neue ersetzt. Dies ist auch beim Internet wieder ganz ähnlich, wovon nicht zuletzt die oben zitierte Prognose des Bill Gates zeugt. Ein Blick zurück auf die einzelnen Innovations- und Entwicklungsschritte der medienkulturellen Evolution in den vergangenen Jahrzehnten aber bezeugt, dass das Neue das Alte nie ersetzt, sondern immer ergänzt und verändert hat. Diese Gesetzmäßigkeit hat Wolfgang Riepl bereits 1913 in seinem Werk über "Das Nachrichtenwesen des Altertums mit besonderer 
Rücksicht auf die Römer" treffend formuliert: Medien werden, ,wenn sie nur einmal eingebürgert und für brauchbar befunden worden sind, auch von den vollkommensten und höchst entwickelten niemals wieder gänzlich und dauerhaft verdrängt und außer Kraft gesetzt [...], sondern sich neben diesen erhalten, nur daß sie genötigt werden, andere Aufgaben und Verwertungsgebiete auszusuchen". Auch in der Netzzeit werden die etablierten Medien weiter existieren, sich aber auch weiter entwickeln müssen. Ob diese Entwicklung in der Konzentration auf Kernkompetenzen liegt, ob sie in der Erschließung neuer Bestandteile der medialen Wertschöpfungskette liegt oder ob sie in der Verbindung von OfflineKonzepten mit der Online-Plattform liegt, wird von folgenden Faktoren abhängen.

\section{Technische Konvergenz und funktionale Differenzierung}

Auch wenn die Komplementarität alter und neuer Medien zur Gesetzmäßigkeit der Medienevolution gehört, muss man für die zukünftige Ausgestaltung des Medienensembles zwischen Technik und Nutzung unterscheiden. So werden die technischen Plattformen für die Verbreitung von Informations- und Unterhaltungsangeboten zunehmend konvergieren. Telefon, Radio, TV und Internet werden in Zukunft in einer technologischen Basisplattform zusammengeführt werden. Über dieses „Multimedia-Terminal" lassen sich alle technischen Dimensionen steuern - die Übertragung von Medienangeboten ebenso wie das Datenmanagement für alle weiteren technischen Anwendungen im häuslichen Lebensbereich ("ubiquitous computing") - die Steuerung der Klimaanlage, der Heizung, des Alarm- und Sicherheitssystems und womöglich auch des selbstorganisierenden Kühlschranks, der über den Anschluss an den Datenhighway Fehlbestände an das nächstliegende Lebensmittellager übermittelt, die dann automatisch - und natürlich auf analogem Wege - nachgeliefert werden.

Demgegenüber werden die Nutzungsformen und -funktionen sich eher ausdifferenzieren. Ausgehend von bisherigen Erkenntnissen über Nutzungsmotive, Nutzungmuster, Gratifikationen und differenzierte Nutzungssituationen im Medienensemble gibt es keinerlei Hinweise darauf, dass sich in diesem Bereich Konvergenzprozesse abzeichnen werden. Die Rezeption eines Hollywoodspielfilms wird weiter unter Bedingungen einer Entspannungssituation (,lean back“), die von beruflichen E-Mails und professionellen Daten weiterhin in einer Arbeitssituation ("lean forward“) stattfinden. Die einzelnen Schnittstellen für Kommunikationsoptionen und Medieninhalte werden daher nicht zusammenwachsen, son- 
dern sich - auf Basis einer gemeinsamen technischen Plattform - weiter ausdifferenzieren.

Ein einfaches Beispiel: Selbst wenn es zukünftig gelingen sollte, elektronisches Papier herzustellen, das sich über den Anschluss an das Internet jeweils aktualisieren lässt, so dass der Nutzer jeden Morgen die aktuelle Tageszeitung über das Netz auf seine persönliche elektronische Zeitung herunterladen kann, so wird dies doch nur dann marktfähig sein, wenn diese elektronische Zeitung so ausgestaltet ist, dass sich das elektronische Papier ähnlich dem herkömmlichen analogen Papier in der U-Bahn, im Flugzeug oder beim Frühstück am Küchentisch auffalten und lesen lässt. Die Tageszeitung hätte damit eine neue technische Plattform erhalten, ihre Inhalte aber blieben tageszeitungsspezifisch, angepasst an zeitungstypische Nutzungsmotive, Nutzungsmuster und Gratifikationen.

\section{Strategische Herausforderungen}

Die beschriebene Verbindung von technischer Konvergenz und funktionaler Differenzierung enthebt Medienunternehmen nicht der Notwendigkeit, Cross-Media-Strategien zu entwickeln, um die jeweiligen Kernkompetenzen in Verbindung mit Markennamen auf allen technischen Plattformen und möglichst vielen Kanälen konsequent nutzbar zu machen (brand buildung, brand extending). Die Strategie „eine Marke - alle Medien" ist aus Anbieterperspektive interessant, weil auf diesem Wege Wertschöpfungsketten verlängert und Zielgruppen konsequenter angesprochen werden können. Aus Nutzerperspektive wiederum eröffnet diese Strategie die Möglichkeit, Glaubwürdigkeits- und Orientierungszuschreibungen, die Rezipientinnen und Rezipienten traditionellen Medienangeboten entgegenbringen, auch auf neue Angebote, beispielsweise im Internet, zu übertragen und sich auf diesem Wege einen Weg durch den "Informationsdschungel" zu bahnen. Eine wichtige strategische Überlegung liegt darin, Netzangebote nicht als Replikation bestehender Offline-Angebote zu konzeptualisieren, sondern die netzspezifischen Bedingungen (Vernetzung, permanente Aktualisierungsmöglichkeit, Unabhängigkeit von Raum- und Zeitbegrenzungen) zu nutzen, um Komplementärangebote mit internetspezifischem Zusatznutzen zu etablieren. Ein Beispiel für diese Strategie liefert die "Financial Times Deutschland". Neben der traditionellen Zeitung bietet der Verlag einen professionell gestalteten Webauftritt, der unter anderem den Zeitungsabonnenten eine kostenlose Archivrecherche der gesamten Offline- und Onlineausgabe ermöglicht. Weiterhin können Abonnenten sich Finanzdaten und anderen Informationen über Handy und Palm-Organizer liefern lassen, so dass jeder Abonnent zusätzlich zur Analogausgabe der Zeitung ein Rundum- 
Informationspaket erhält, das die verschiedenen technischen Plattfor. men mit ihren spezifischen Informationssegmenten konsequent nutzt.

\section{3. Ökonomische Konsequenzen}

Eine wesentliche Zukunftsentscheidung für Medienanbieter, beispielsweise für Verlage, wird darin liegen, wie die zusätzlichen Angebote im Netz eigentlich finanziert werden sollen. Dabei sind vor allem zwei Probleme zu lösen: Zum einen zeigt die Entwicklung bei den Einkünften der Online-Medien, dass es bisher nur einem überaus geringen Teil der Anbieter gelingt, zusätzliche Netzangebote über Abonnementgebühren zu finanzieren. Dies ist vor allem für die Anbieter problematisch, die qualitativ hochwerte Angebote ins Netz stellen. Außerhalb des Netzes müssten sie bezahlt werden, im Internet aber werden sie kostenlos angeboten. Nach der Strategie „Dabei sein ist alles" haben Verlage lange Zeit teure und hochwertige Angebote kostenlos ins Netz gestellt, um nicht den Anschluss an die Online-Entwicklungen zu verlieren. Bislang zeigt sich allerdings, dass Werbung die wesentliche Finanzierungsquelle für Online-Angebote ist und wohl auch zukünftig bleiben wird. Nur sehr spezifische Zusatzangebote lassen sich über Abonnementgebühren refinanzieren (beispielsweise professionelle Datenbankrecherche).

Dementsprechend gilt es gerade für Medienunternehmen, die Trennung zwischen Werbung und redaktionellen Inhalten genau $\mathrm{zu}$ beachten. Wenn Werbung auch zukünftig die wesentliche Finanzierungsstrategie für Online-Angebote von Medienunternehmen bleibt, so müssen Informationsanbieter damit rechnen, dass auch Netznutzer sich professionalisieren und emanzipieren. Die Trennung zwischen Werbung und redaktionellen Inhalten wird also zukünftig genauer beachtet werden und über die Glaubwürdigkeit und Qualität eines Angebots entscheiden. Links zu Anzeigenkunden in redaktionellen Artikeln waren lange Zeit verlockend, sind langfristig allerdings verderblich. Dies haben verschiedene OnlineAnbieter, beispielsweise Spiegel-Online, längst erkannt und entsprechend reagiert. Medienunternehmen werden darüber hinaus neue $\mathrm{Ge}$ schäftsmodelle entwickeln müssen, über die traditionelle Offline-Inhalte mit neuen Online-Inhalten kombiniert werden können, beispielsweise über gestaffelte Abonnementgebühren, je nach Informations- und Angebotsspektrum. Die Möglichkeiten der Internetkommunikation werden allerdings auch manche herkömmlichen Strukturen in Frage stellen. Ein Beispiel dafür liefert die Onlinestrategie des US-amerikanischen Erfolgsautors Stephen King. Er vermarktete seine beiden aktuellen Erzählungen über das Internet. So bot King seine im März 2000 erschienene Erzählung „Riding the Bullet" nur über das Internet an. Gegen Zahlung 
von 2,5 US-Dollar konnten seine Fans sich die Geschichte herunterladen. Nur zwei Tage hat es gedauert, bis mehr als 500.000 Fans die Geschichte im Netz gekauft und heruntergeladen hatten. Stephen King verdiente innerhalb von diesen zwei Tagen mehr als eine Million US-Dollar. Es dauerte allerdings auch nicht mehr als zwei Tage, bis findige Fans die Verschlüsselung geknackt hatten, um die Geschichte ohne Bezahlung herunterzuladen. Dem Verlag Simon \& Schuster Online blieb nichts anderes übrig, als die Geschichte nach zwei Tagen offen zugänglich ins Netz zu stellen, da der Code ohnehin bekannt war. Die Vermarktung im Netz kann also große - auch finanzielle - Erfolge zeitigen, diese sind allerdings oftmals ebenso schnell vorüber, wie sie begonnen haben.

Der zweite Versuch Stephen Kings bietet noch interessantere Perspektiven: Seine neueste Publikation "The Plant" vermarktete King häppchenweise über das Internet. Das erste Kapitel stellte der Autor kostenlos ins Netz - allerdings unter der Maßgabe, dass eine Fortsetzung der Geschichte nur dann geschrieben würde, wenn mehr als 75 Prozent aller Leser freiwillig einen US-Dollar zahlten. Die Rechnung ging auf: Genau 76,38 Prozent aller Leser zahlten freiwillig einen Dollar, und Stephen King schrieb weitere Kapitel. Mit jedem Kapitel allerdings nahm die Zahlungsmoral der lesewilligen Fans ab, so dass King nach dem fünften Kapitel die Geschichte einstellte. Auch dies ist ein Beleg dafür, dass im Internet Vermarktungsstrategien kurzfristig, aber nicht unbedingt langfristig erfolgreich sein müssen. Außerdem koppelte King mit seiner Internetvermarktungsstrategie als Autor den Verlag als Schnittstelle zwischen Schreiber und Leser aus. Diese Formen des Direktmarketings ohne Zwischenstationen werden eine weitere zukünftige Herausforderung für Medienunternehmen darstellen.

\section{Qualitative Herausforderungen}

Eine überaus spannende Frage liegt in der zukünftigen Ausgestaltung von Inhalten im Medienensemble. So wurde zu Beginn des Vernetzungsprozesses wild über die Innovationspotentiale des Online-Journalismus spekuliert. Inzwischen wissen wir, dass Qualitätskriterien sich im Netz nicht grundsätzlich von denen außerhalb des Netzes unterscheiden. Betrachtet man beispielsweise die Entwicklung der page impressions im Segment der redaktionellen General-Interest-Angebote, so zeigt sich sehr schnell, dass die Menschen im Netz ähnliche Nutzungsmuster und Nutzungspräferenzen offenbaren wie außerhalb des Netzes. Dies lässt sich relativ leicht erklären: Medienangebote, die sich offline Kompetenzen und ein entsprechendes Image aufgebaut haben, haben damit auch in der Netzkommunikation einen erheblichen Vorteil. Die Glaubwürdig- 
keitszuweisungen durch Rezipientinnen und Rezipienten vollzieht sich nämlich gerade bei der Informationsnutzung im Netz im Wesentlichen nach bekannten Parametern, die netzextern etabliert worden sind. Für Medienanbieter ist es unter Qualitäts- und Glaubwürdigkeitsgesichtspunkten also wesentlich, dass ein Imagetransfer von den Offline- zu den Online-Angeboten erfolgt, um darauf bei der Netzkommunikation aufbauen zu können.

Die Qualitätsstrategie von Medienunternehmen im Online-Zeitalter lässt sich daher - ein wenig überspitzt - mit einem "weiter so" beschreiben. Dieses „weiter so“ (GO ON) setzt im Einzelnen folgende strategische Überlegungen voraus:

\section{- Glaubwürdigkeit}

Durch einen entsprechenden Image-Transfer lassen sich außerhalb des Netzes erworbene Glaubwürdigkeitszuschreibungen in das Internet transferieren. Gerade angesichts der Informationsflut, die im Netz von jedem Nutzer bewältigt werden muss, ist die Glaubwürdigkeit von Medieninhalten eine sichere Bank, von der aus sich auch im Internet agieren lässt.

\section{- Orientierung}

Rezipientinnen und Rezipienten benötigen gerade im Internet Orientierungshilfen, um diese Informationsflut zu bewältigen. Auch diese Orientierungen können eher etablierte Medienanbieter geben, weil sie netzextern auf eine entsprechende Tradition verweisen können und auch diese ins Internet überführen können. Gerade weil im Netz viele Informationen ohne Quellenangabe angeboten werden, Nutzerinnen und Nutzer also nicht validieren können, inwiefern es sich um verlässliche Informationen handelt, haben etablierte Anbieter einen erheblichen Marktvorteil.

\section{- Originalität (Mehrwert)}

Das Gebot der Komplementarität von Inhalten und Informationsstrategien verlangt von Medienanbietern, im Netz einen Zusatznutzen zu implementieren, einen informatorischen Mehrwert, der beispielsweise über Exklusivität von Informationen, über Aktualitätsgebundenheit oder über Orientierungsleistungen im Sinne von umfassenden Hintergrundinformationen gelingen kann.

\section{- Nähe}

Auch und gerade in der anonymen Netzkommunikation ist es notwendig, eine emotionale Ansprache der Rezipienten zu gewährleisten. Es geht

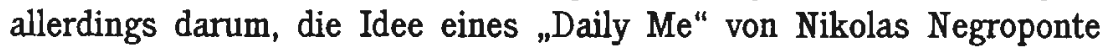


anders umzusetzen, als er es angedacht hat: Jedes Medienangebot muss für die Nutzer jeweils als Daily Me interpretierbar sein, also zielgruppenspezifisch ausgerichtet und gestaltet sein. Nikolas Negroponte hat allerdings etwas anderes gemeint: Er geht davon aus, dass Nutzerinnen und Nutzer in Zukunft über digitale Agenten (,personal digital agents") sich abhängig von ihrer jeweiligen Interessensspezifikation alltäglich ihr persönliches Medienangebot zusammenstellen lassen. Womöglich wird es genau umgekehrt sein: Der Vorteil der traditionellen Medienkommunikation liegt gerade darin, dass ein informatorisches Rundumangebot der Tageszeitung oder ein Vollprogramm im Fernsehen deshalb überzeugt, weil der Nutzer sich vom Angebot noch überraschen lassen kann. Wer sein individuelles Informationsprofil so festgelegt hat, dass er auch nur zu diesen jeweiligen Themengebieten Informationen zusammengestellt und geliefert bekommt, dem wird es kaum noch gelingen, sich durch die Angebote überraschen $z u$ lassen. Gerade darin mag der Vorteil einer Verbindung von traditionellen und innovativen Medienangeboten über das Internet liegen. Die Überzeugungskraft und das Qualitätsimage des jeweiligen Mediums liegt auch darin, dass die Anbieter in der Lage sind, die Nutzer durch Akzentuierungen und hintergründige Ausgestaltungen zu überraschen. Dies wird gerade in Zeiten neuer notwendiger Orientierung von Bedeutung sein.

Nicht zuletzt wird die Qualität und Marktfähigkeit von Angeboten im Internet auch davon abhängen, ob die Anbieter in der Lage sind, neue Gemeinschaften strategisch zu erschließen. Mehr als jede andere Kommunikationsplattform ermöglicht das Internet zielgruppenspezifisches Informations- und Kommunikationsmanagement. Wenn für das Netz nicht mehr von Zielgruppen, sondern von "communities of interest", "communities of choice" oder auch "communities of believe" die Rede ist, so eröffnen sich dadurch neue soziale Vernetzungspotentiale, die gerade angesichts der Technologisierung unserer Gesellschaft häufig in Frage gestellt werden. Glaubensgemeinschaften, die es beispielsweise zunehmend schwer haben, ihre Mitglieder für eine aktive Beteiligung zu motivieren, können an dieser Stelle ansetzen. Über Kommunikationsstrategien lässt sich gerade in diesem Bereich ein "gemeinschaftlicher Mehrwert" etablieren, der ohne die Vorteile der Netzkommunikation schwer zu implementieren ist. Wenn eine allmorgendlich gesendete SMS-Botschaft (zum Beispiel ein Bibelspruch) dazu beitragen kann, das Zugehörigkeitsgefühl von Menschen zueinander zu stärken, so ist dies eine von vielen Optionen, die die Netzkommunikation bereithält und die jenseits der streng marktwirtschaftlichen Betrachtungen des Internet belegen, dass es immer eine Frage der Perspektive ist, ob neue Technologien auch für traditionelle Einsatzgebiete erschlossen werden können. 


\section{Ausblick: Evolution oder Revolution?}

Es ist also keine Medienrevolution, die durch das Internet angestoßen worden ist oder uns noch bevorsteht. Vielmehr fügen sich das Netz und die Netzkommunikation in einen medienkulturellen Evolutionsprozess ein, der auch heute wieder durch ähnliche Indikatoren und Konstanten geprägt wird, wie dies schon immer der Fall war. Es wird uns kein plötz. licher und dramatischer Umbruch der gesellschaftlichen Kommunikationsverhältnisse bevorstehen, sondern wir befinden uns in einem kontinuierlichen und langfristigen Adaptionsprozess an zum Teil sicherlich grundlegend neue Verhältnisse der Netzwerkkommunikation. Selbst wenn dies keine Revolution bedeutet, so ist doch eines zu berücksichtigen: Auch Evolution bringt von Zeit zu Zeit radikale Veränderungen mit sich. So mussten die Dinosaurier sterben, weil sie nicht mehr anpassungsfähig, im Evolutionsprozess also nicht mehr anschlussfähig waren. Wer sich in der Reorientierung des Medienensembles als Dinosaurier erweist, wird langfristig keine Chancen haben. Wer sich strategischevolutionär an Kombinationen von traditionellen und innovativen Medienangeboten, von Offline- und Online-Strategien heranwagt, wird langfristig lebendiger und aktiver Bestandteil des Medienensembles bleiben. ${ }^{2}$

2 Als weiterführende Literatur sei zum Thema empfohlen: Miriam Meckel:: Die Medien-Matrix. Konturen und Schnittstellen des Computerzeitalters, in: Miriam Meckel/Marianne Ravenstein ( $\mathrm{Hg}$.): Cyberworlds. Computerwelten der Zukunft, Ottobrunn 2000, 9-36. Christoph Neuberger/Jan Tonnemacher (Hg.): Online - Die Zukunft der Zeitung? Das Engagement deutscher Tageszeitungen im Internet, Opladen-Wiesbaden 1999. Wolfgang Riepl: Das Nachrichtenwesen des Altertums mit besonderer Rücksicht auf die Römer, Leipzig u.a. 1913. 